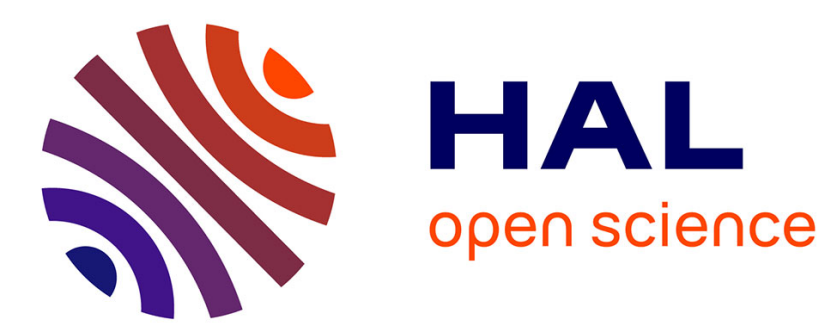

\title{
Les chênaies des montagnes pyrénéo-cantabriques, un élément forestier du système agro-pastoral Jean-Paul Métailié
}

\section{To cite this version:}

Jean-Paul Métailié. Les chênaies des montagnes pyrénéo-cantabriques, un élément forestier du système agro-pastoral. Revue Géographique des Pyrénées et du Sud-Ouest, 1986, 57 (3), pp.313-324. 10.3406/rgpso.1986.4950 . hal-01362879

\section{HAL Id: hal-01362879 \\ https://hal-univ-tlse2.archives-ouvertes.fr/hal-01362879}

Submitted on 9 Sep 2016

HAL is a multi-disciplinary open access archive for the deposit and dissemination of scientific research documents, whether they are published or not. The documents may come from teaching and research institutions in France or abroad, or from public or private research centers.
L'archive ouverte pluridisciplinaire HAL, est destinée au dépôt et à la diffusion de documents scientifiques de niveau recherche, publiés ou non, émanant des établissements d'enseignement et de recherche français ou étrangers, des laboratoires publics ou privés. 
Les chênaies des montagnes pyrénéo-cantabriques, un élément forestier du système agro-pastoral

Jean-Paul Métailié

\section{Citer ce document / Cite this document :}

Métailié Jean-Paul. Les chênaies des montagnes pyrénéo-cantabriques, un élément forestier du système agro-pastoral. In: Revue géographique des Pyrénées et du Sud-Ouest, tome 57, fascicule 3, 1986. L'élément et le système. pp. 313-324. doi : 10.3406/rgpso.1986.4950

http://www.persee.fr/doc/rgpso_0035-3221_1986_num_57_3_4950

Document généré le 28/01/2016 


\title{
Resumen
}

Los robledales en las sierras pirineo-cantábricas : elemento forestal del sistema agropastoril. En las sierras pirineo-cantábricas, los robledales son un indicador de las relaciones entre el bosque y la sociedad de montaña. Fueron el foco del espacio rural en el que se concentraron los aprovechamientos más diversos, tanto pastoriles como forestales. Víctimas de la explotación tradicional (pastoreo intensivo, quemadas) que los ha alterado profundamente, a la vez fueron protegidos cuidadosamente como inidspensable reserva de madera. Hoy los desequilibrios dominan: el robledal aparece sea como un fósil silvo-pastoril, sea como una forma transitoria hacia el yermo.

\section{Résumé}

Les chênaies constituent dans les montagnes pyrénéo-cantabriques un révélateur des relations entre forêt et société montagnarde. Elles sont le maillon de l'espace villageois où se sont concentrés les usages les plus variés, tant pastoraux que forestiers. Ces peuplements furent à la fois victimes de l'exploitation traditionnelle (pastoralisme intense, pratique du feu), qui les a profondément remodelés, et soigneusement protégés en tant que réserve de bois indispensable. Aujourd'hui les déséquilibres sont généraux, la chênaie représentant soit un fossile sylvo-pastoral, soit une forme d'enfrichement transitoire.

\begin{abstract}
Oak groves in the Pyrenees and cantabrio mountains. A forestry component of the agro-pastoral system. In the Pyrenees and Cantabric mountains oak woods can be considered as a link between the forest and the mountain society. They are the spot of the village scenery where are seen together diverse uses, as much pastoral as forest, by natives. These plantations are victims of traditional exploitation (intensive pastoralism, firing) which have deeply changed them, whilst being protected as an indispensable wood reserve. Presently disequilibrium is general, the plantations being either a sylvipastoral fossil, or a transitory fallow land.
\end{abstract}


REVUE GÉOGRAPHIQUE DIES PYRÉNÉES ET DU SUU-OUIS'T' TOME 57, Fasc. 3, pp. 313-324, Toulouse, 1986.

\title{
Les chênaies des montagnes pyrénéo-cantabriques, un élément forestier du système agro-pastoral
}

\author{
par Jean.Paul Métailié *
}

\begin{abstract}
Les relations entre les sociétés agro-pastorales pyrénéennes et leur forêt ont longtemps été réduites à une attitude de prédation pure et simple. Les images du paysan-ennemi-de-l'arbre, du berger pyromane, étaient véhiculées par la plupart des auteurs jusqu'à une date récente... L'analyse historique, la réinterprétation de la dynamique des formations végétales et de leur succession, permettent de mieux définir les rapports entre les sociétés montagnardes et leurs forêts. La forêt paysanne apparaît comme une formation typique par sa physionomie et par son histoire, longtemps laissée à l'écart des recherches peutêtre à cause de son aspect rébarbatif et très peu «forestier ». Le chêne s'individualise comme un des éléments majeurs de cette forêt et peut former dans certaines vallées des peuplements originaux (1).
\end{abstract}

\section{Une forêt diagonale}

La place de la chênaie dans l'étagement de la végétation en montagne est tout à fait classique du schéma bioclimatique (2). Dans les

\footnotetext{
* Chargé de recherche au CNRS, UA 366, Institut de géographie, Université de Toulouse-Le Mirail, 31058 Toulouse cedex.

(1) J.-P. MÉTAiLIÉ, La forêt paysanne dans les Pyrénćes centrales, RGPSO, $55,2,1984$, pp. 231-238.

(2) M. Gruber, La chênaie rouvre dans les Pyrénées ariégeoises. Bull. Soc. Bot. Fr. 120, 1973, pp. 123-132. M. GRUBER, Le chêne sessile dans la vallée du Louron. Bull. Soc. Hist. Nat. Toulouse., 1981, pp. 165-174. R. NËGR (1972), La végétation du bassin de l'One (Pyrénées centrales); $4^{\mathbf{e}}$ note: les forêts. Veröff. Geobot. Inst. Rübel, 49, 1972, pp. 1-125.
} 
Pyrénées centrales, où la masse de la hêtraie-sapinière humide est l'élément dominant du paysage forestier, les chênaies dessinent avec précision les situations d'abri, les expositions les plus sèches et ensoleillées. Chaque espèce de chêne correspond à un étage bioclimatique : Quercus ilex dans les bassins à tendance sub-méditerranéenne, $Q$. pubescens et $Q$. pedunculata sur les collines de l'avant-chaîne et dans les basses vallées, $Q$. petraea coiffant le tout dans les hautes vallées mais se retrouvant aussi bien sur le piémont. Dans les montagnes cantabriques centrales, le dispositif, tout en étant similaire, est compliqué par des gradients plus courts (opposition entre versant atlantique et versant castillan, forte situation d'abri en Liébana avec présence de $Q$. suber), et par l'abondance du chêne tauzin ( $Q$. pyrenaica) (3).

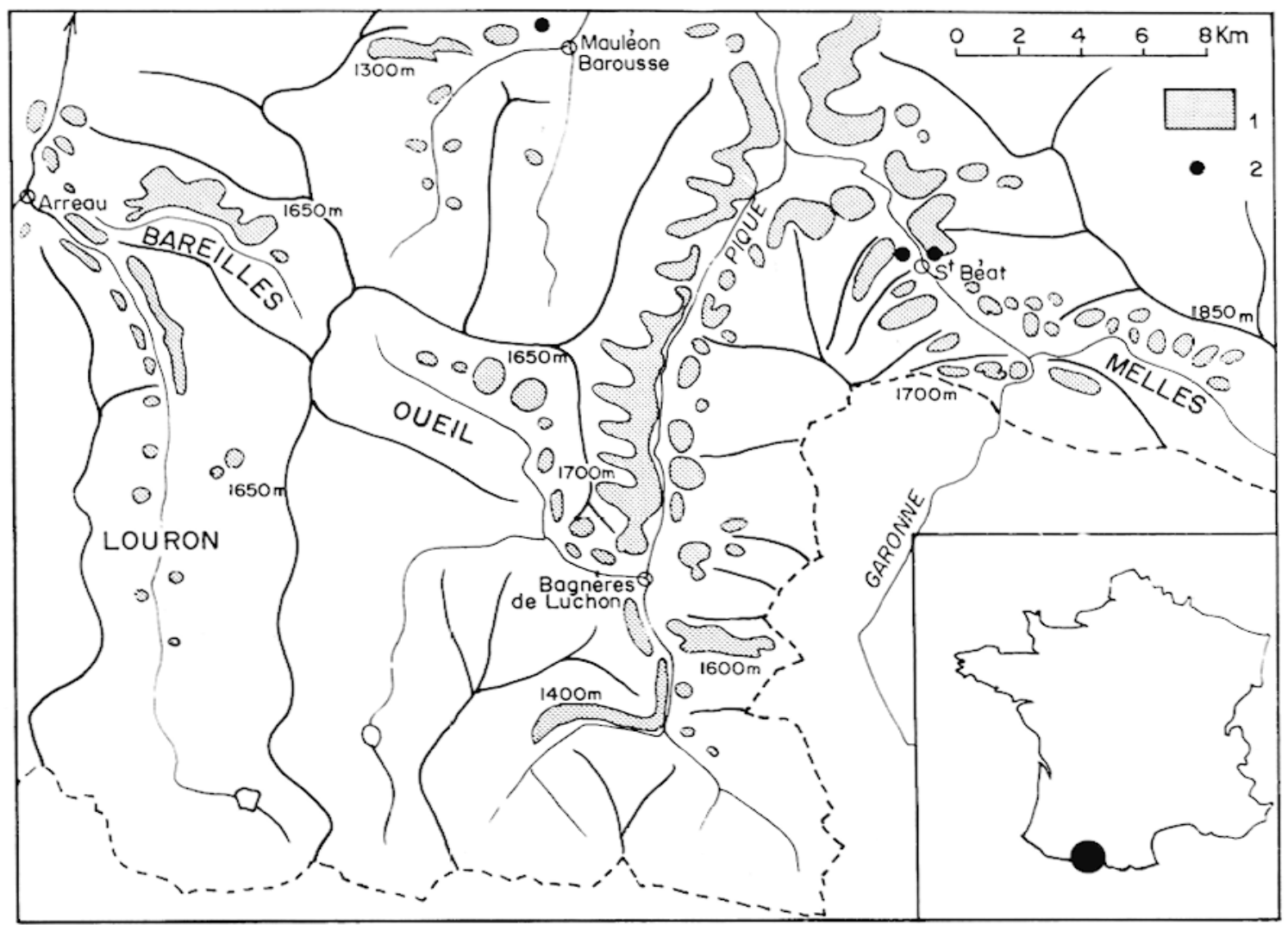

FIG. 1

Localisation des chênaies montagnardes dans les Pyrénées centrales.

1. Chênaic à Quercus petraea. - 2. Station à Quercus ilex. Les altitudes indiquées aux cotes maximales atteintes par Quercus petraea.

(3) Chêne sessile $=$ Quercus sessiliflora Salisb. $=$ Q. petraca (M. Liebl.) Chêne pédonculé $=$ Rouvre $=\mathrm{Q}$. pedunculata Ehrh. $=\mathrm{Q}$. robur $\mathrm{L}$. - Chêne tauzin $=$ Q. Toza Bosc. $=$ Q. pyrenaïca Will. Cf. également B. Becker. Quelques observation morphologiques chez le Chêne sessile et le Chêne pédonculé. Bull. Soc. Bot. Fr., 119, 1972, pp. 231-236. 
Située dans les secteurs les plus favorables à l'activité humaine en montagne, la chênaie a été quasiment détruite par les défrichements; il n'en reste plus aujourd'hui que des lambeaux discontinus. Pourtant, l'aptitude écologique d'espèces comme $Q$. petræa dans les Pyrénées françaises ou $Q$. pyrenaica dans les montagnes cantabriques est extrêmement large et permet de trouver des chênaies dans les endroits les plus inattendus. Dans le haut bassin de ia Garonne (Nestes, Pique, Garonne), des peuplements s'étendent du fond dés vallées jusqu'à des altitudes très élevées : 1500 à $1650 \mathrm{~m}$ dans le Louron (Bareilles, Mont); $1750 \mathrm{~m}$ dans la vallée de Couplan (Aure); $1650 \mathrm{~m}$ en vallée d'Oueil; $1700 \mathrm{~m}$ sur les flancs est du massif du Burat et jusqu'à 1 800-1850 $\mathrm{m}$ dans la vallée de Melles. Nulle part ailleurs dans les Pyrénées françaises le $Q$. petrcea ne monte plus haut : il ne dépasse pas 1500-1600 m dans le Vicdessos ou la vallée de l'Aston (en Ariège), $1500 \mathrm{~m}$ dans la vallée de Cauterets (pic de Viscos) et n'atteint guère que $1450 \mathrm{~m}$ près de Mont-Louis, en Cerdagne (4) (fig. 1 ).

Le chêne sessile arrive donc à coiffer la plupart des séries dè végétation de l'étage forestier montagnard; son cortège floristique présente d'ailleurs de fréquentes affinités avec celui des hêtraies-sapinières: abondance de Vaccinium myrtillus, Prenanthes purpurea, Asperula odorata, Deschampsia flexuosa, Mercurialis perennis, etc. Les régénérations de hêtres ou de sapins $y$ sont nombreuses, préfigurant parfois une évolution de la composition du peuplement. La chênaie de Melles, haute vallée de la Garonne, culmine même sous la forme d'une étonnante "garrisade " sub-alpine accrochée aux rocheís de la paroi, entourée d'une végétation où dominent Cytisus purgans, Festuca eskia, Festuca scoparia, Trifolium alpinum, etc. (soulane de Labach) (fig. 2).

Dans les montagnes cantabriques centrales, le chêne tauzin (qui n'a rien ici du tauzin eu-atlantique des plaines de la façade atlantique française) se comporte de la même façon que le chêne sessile dans les Pyrénćes. Les altitudes atteintes ne sont pas aussi ćlevées qu'en France mais comparativement tout aussi spectaculaires : le caractère océanique accentué du massif "écrase " les étages de végétation et rabaisse la limite inférieure du «sub-alpin " entre $1400 \mathrm{~m}$ et $1600 \mathrm{~m}$. Malgré cela, on trouve le tauzin jusqu'à $1500 \mathrm{~m}$ dans le val de Prioro. $1450 \mathrm{~m}$ de part et d'autre du col de Piedras Luengas (Liébana et vallée de Pisuerga) et jusqu'à $1600 \mathrm{~m}$ dans la Tierra de la Reina, sur le versant sud du Puerto de San Glorio. En Liébana, les chênes s'étendent sur les moindres soulanes, montant jusque vers $1400 \mathrm{~m} \mathrm{(5).}$

Ce chevauchement des étages de végétation doit être interprété. Si le chêne accompagne fidèlement les situations d'abri et les soulanes. celles-ci correspondent aussi aux lieux privilégiés de l'occupation

(4) Observations personnelles et H. GAUSSEN, Les forêts de la vallée d'Aure, RGPSO, 2, 1931, pp. 241-250.

(5) G. Bertrand, Apogée et déclin d'un géosystème sylvo-pastoral (Montagnes de Léon et de Palencia, Espagne du Nord-Ouest, RGPSO, 55, 2, 1984, pp. 239-248. 


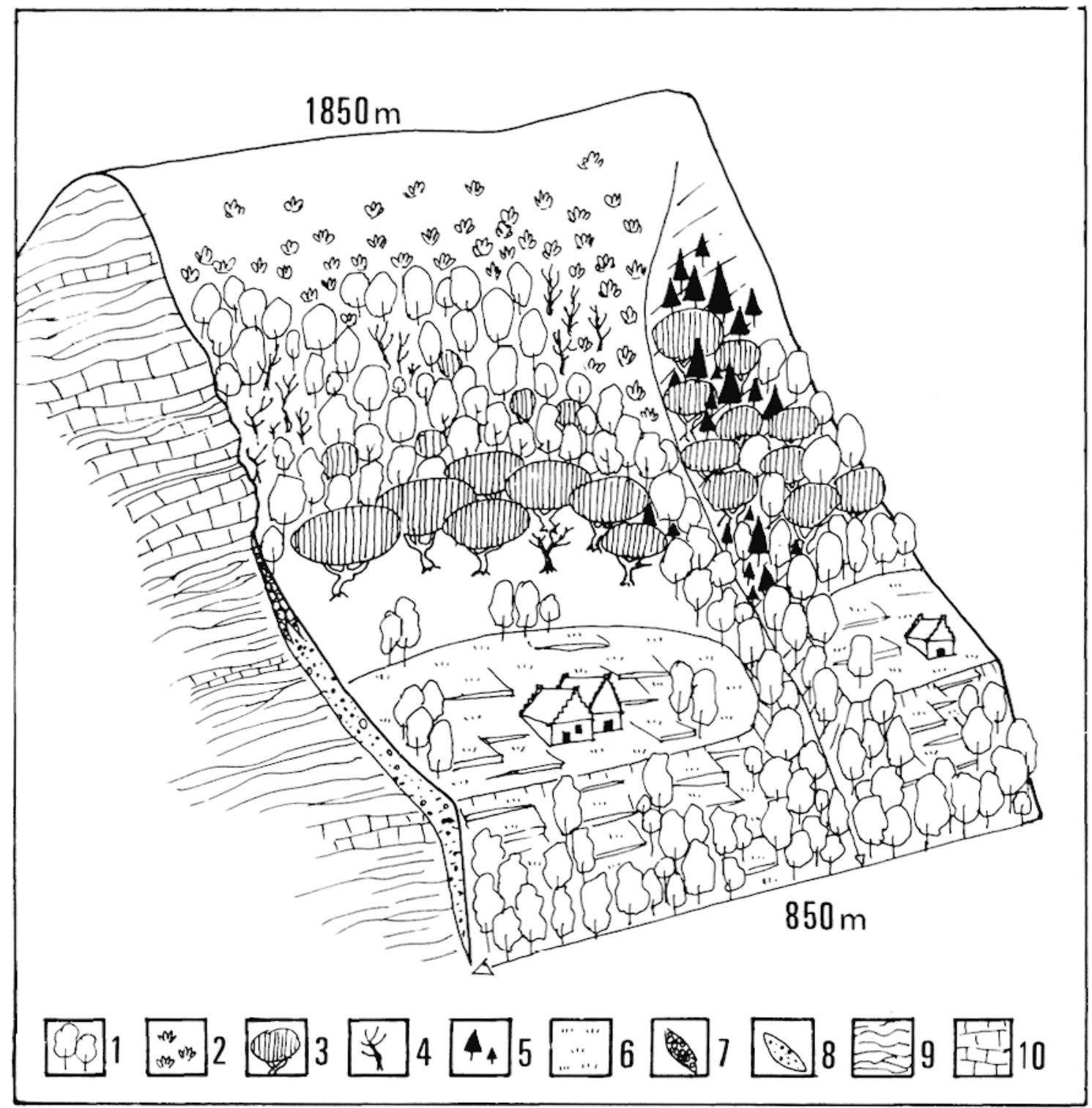

Fig. 2

Organisation des milieux sur la Soulane de Labach de Melles.

1. Chênaie. -2 . Brousse à chêne. - -3 . Hêtraie (arbres têtards, souches recépées). - 4. Arbres brûlés. - 5. Sapins et semis de sapins. - 6. Prairies et pâtures privées. - 7. Dépôts de versant grossiers. - 8. Dépôts de versant fins et moraines. - 9. Schistes. - 10. Calcschistes.

humaine, et cela depuis fort longtemps : entre 2000 et 3800 BP dans les Pyrénées centrales (6). La référence au climax serait ici dogmatique; le chêne est bien un élément du climax, mais la chênaie ressortit au moins autant à l'histoire; c'est un fait pastoral, ou plutôt paysan, dans son extension, sa physionomie et sa dynamique.

(6) G. Jalut, L'action de l'homme sur la forêt montagnarde des Pyrénées ariégeoises et orientales depuis 4000 BP d'après l'analyse pollinique, Actes $106^{\circ}$ Congrès Nat. Soc. Sav. 1981, pp. 163-174. 


\section{La chênaie, maillon essentiel de l'économie paysanns}

\section{Une forêt pastorale.}

Dans les Pyrénées centrales, les chênaies sont en général à proximité des villages, enclavées dans les pâturages de demi-saison. Ces versants de soulane déneigent très vite et très souvent en hiver, ne restant inaccessibles que pendant de courtes périodes: les troupeaux y sont conduits aussi souvent que possible pour ménager lés réserves de fourrage. Les chênaies de soulane sont pâturées, tout comme les herbages aux alentours; il s'agit de taillis bas et clairsemés qui permettent le développement d'une strate herbacée souvent aussi dense et de la même composition que dans le vacant (pelouse à Brachypodium, Avena, Lathyrus, Deschampsia, Anthoxanthum, etc.). Cette pelouse de sous-bois s'enfriche rapidement, laissant la place à des fourrés à Sarothamnus scoparius et Pteridium aquilinum sur sols profonds, avec Calluna vulgaris et Genista pilosa quand le substrat devient rocailleux et plus acide. Les vaccinaies à $V$. myrtillus occupent les secteurs les plus ombreux et humides. accompagnant les chênaies les mieux développées et mélangées de hêtres.

Cet enfrichement spontané est combatı par le feu, de la même manière que dans les pâturages voisins : pratique encore vivante dans certaines vallées, celle de la Garonne en particulier. L.es chênaies des Arguts et de Fos ont, par exemple, été incendićes en 1983, 1984 et 1985; les incendies sont fréquents aussi à Melles. Lors de la sécheresse d'avril 1984, on a pu voir des incendies dans de nombreuses chênaies: les versants sud-est du Burat, entre autres, ont été parcourus par des feux amorcés dans le fond de la vallée et qui ont fidélement suivi les peuplements de chênes jusqu'à leur limite, à $1600 \mathrm{~m}$ d'altitude (pic de Sacaube); les incendies passaient aussi dans les hêtraies claires mais débordaient peu dans les futaies de hêtres et sapins. Pendant la sécheresse de l'automne 1985, nombre de chênaies furent touchées par des feux volontaires ou accidentels, souvent violents; l'absence de pluies pendant près de trois mois. desséchant les sous-bois et homogénéisant les contacts entre les divers peuplements, a permis le passage de l'incendie dans des secteurs qui n'étaient plus brûlés depuis longtemps; ce fut le cas sur la rive gauche de la Pique, entre Luchon et Antignac, où des chênaies en voie de recolonisation par le sapin ont été parcourues par un feu échappé d'un vacant.

Il est évident que l'incendie, surajouté au broûtage constant des jeunes sujets, est un très puissant moven de sélection des esnèces et de modelage de la forêt pastorale. Les sapins, très combustibles, à l'écorce fine et ne rejetant pas de souche, sont les premiers éliminés par disparition de toute régénération dans un temps très court; les hêtres résistent à une certaine intensité du feu, grâce à leur capacité de régénération végétative et à leur résistance aux blessurcs, mais 
au-delà d'une périodicité d'une quinzaine d'années ils disparaissent aussi du fait de la destruction des régénérations, des blessures de plus en plus profondes et de l'épuisement des souches. Seul le chêne, protégé par une écorce épaisse, rejetant presque indéfiniment de souche, peut survivre à une fréquence élevée d'incendies. Mais le feu impose alors une physionomie qui n'a plus rien de forestière : arbres aux formes torturées, avec de profondes blessures, formant un taillis bas de recépage, voire, dans les cas ultimes, une brousse de un à deux mètres de hauteur parsemée de troncs noircis, paysage d'allure méditérranéenne propulsé en haute montagne (Melles). La perpétuation du processus conduit à la formation d'un pâturage parsemé de quelques cépées s'épuisant petit à petit.

L'évolution fut identique dans les montagnes cantabriques, mais amplifiée à l'extrême par l'absence de toute prolection de la forếi jusqu'à une date récente et surtout par le poids de la Mesta sur le versant sud. Les centaines de milliers de "merinas " qui venaient pâturer chaque année sur les "puertos pirenaicos " utilisaient autant les forêts que les landes. Les boisements qui subsistent aujourd'hui sur le versant castillan, après des siècles de surpâturage et d'incendies, ne sont plus que des brousses plus ou moins denses (Pisuerga, Tierra de la Reina) ou au mieux des forêts-parcs (Prioro) piquetées de vieilles souches dépérissantes (photo 2). Dans bien des cas l'ancienneté du pastoralisme et des pratiques du feu a provoqué le franchissemeni de seuils peut-être irréversibles : carbonisation des sols, assèchement des versants et épuisement des souches semblent se conjuguer pour perpétuer, sur le versant sud des montagnes cantabriques, un paysage de maigres taillis clairsemés, d'autant plus que le hêtre y est peu dynamique. Une telle situation est plus rare en France, en raison de l'exiguïté de la plupart des chênaies et de la présence d'espèces colonisatrices.

La chênaie pastorale n'est pas seulement modelée pour les troupeaux d'ovins et de bovins et par eux. Aussi très précieuse pour lout le "menu bétail », elle nourrit en particulier les porcs des villages, qui étaient rassemblés en troupeau et menés à la glandée par un porcher communal. Saint-Bertrand de Comminges, à la fin du XVIII' siècle, formait chaque jour un troupeau d'une centaine de porcs, conduit dans les forêts des collines avoisinantes. A Bareilles, vers $1870-1880$, c'étaient 50 à 100 porcs qui utilisaient les chênaies de la soulane, en sus des 300 à 500 bovirıs, 1500 ovins et 50 bêtes de somme, ce qui pour 300 à 400 ha de forêts déclarés défensables chaque année, donnait une charge animale en forêt considérable (7).

L'abondance de $Q$. pedunculata dans les vallées peut être reliée à sa grande productivité en glands; le chêne Rouvre était planté en haies bocagères d'où il a essaimé depuis quelques décennies. Le Rouvre

(7) Arch. munic. Saint-Bertrand, série BB3. - AD 65, Carnets journaliers des gardes forestiers (Bareilles). 
était probablement le seul arbre du monde paysan à ne pas être émondé (pour préserver la glandée) et il forme toujours de grands arbres se distinguant des sessiles voisins.

Enfin, dernier utilisateur de la chênaie, et non des moindres, la chèvre permettait de rentabiliser le pâturage vertical que constituent les taillis bas, bouclant ainsi les possibilités d'utilisation de la chênaie. A peu près éliminée au XIX" siècle dans les Pyrénées françaises, elle garde une grande importance dans les montagnes cantabriques.

\section{Une banque paysanne.}

Forêt pastorale, revendiquée comme telle, exploitée souvent sans limite, la chênaie est aussi une réserve de bois indispensable que les communautés ont su conserver et protéger, y compris contre les projets d'aménagement des forestiers. Le bois de chêne, dur et non cassant, servait pour la construction des charpentes (à une époque où les incendies étaient fréquents) et pour la confection d'outils et d'instruments aratoires; c'était aussi une réserve de bois de feu de secours utilisé lors des hivers exceptionnels. Peu étendues, localisées, les chênaies de qualité furent soigneusement préservées dans les Pyrénées françaises.

"Le chêne est très recherché en Oueil en raison de sa rareté ", affirme en 1890 l'inspecteur des Eaux et Forêts de Luchon, alors même qu'il est en train de préparer un plan d'aménagement fondé sur le recépage en hêtraie des bois de chêne "dégradés ". On touche là une des nombreuses oppositions irréductibles entre forestiers et paysans, portant sur l'appréciation de la qualité des peuplements. Pour les forestiers, qui recherchent la meilleure production commerciale, le chêne est ici sans valeur, comparé aux peuplements du centre et du nord de la France; sapin et hêtre restent les produits forestiers locaux les plus rentables. On aboutit ainsi à des situations paradoxales, comme à Melles, où les habitants du hameau de Labach, par ailleurs qualifiés de "délinquants ataviques", en viennent, en 1890 à refuser pendant quatre années successives l'affouage que les forestiers leur proposaient dans les chênaies toutes proches $(200 \mathrm{~m})$, préférant exploiter les hêtraies du versant opposé, au prix d'un long trajet; la pétition des habitants invoque l'utilité vitale du chêne comme bois de charpente; en 1763, déjà, un règlement communal de Melles interdisait la coupe des chênes, sauf pour usage des particuliers et sur permission des consuls (8).

Près de Luchon, la commune de Sode s'oppose également, en 1849. à l'exploitation de la chênaie proche du village. Cette essence rare. déclare le Conseil municipal, est indispensable pour la charpente. son gland nourrit les cochons et les branches peuvent être émondées

(8) Arch. munic. Melles. 
si nécessaire : les villageois demandent que l'on coupe à la place les hêtres subsistant dans le bois en question (9).

On pourrait accumuler ces exemples. Il date dune époque où la forêt commence à être étroitement surveillée par les gardes forestiers qui imposent leur mode de gestion, restreignant la liberté d'usage des paysans. Ils restent révélateurs de l'action sélective qui s'est exercée pendant plusieurs siècles sur les peuplements.

Il est possible d'imaginer des peuplements avant intervention humaine très mélangés (chênes-hêtre-sapin avec tilleuls, ormes, bouleaux. etc.), y compris sur les soulanes. Plusieurs siècles ou millénaires de pacage, incendies, coupes, sélection volontaire, ont conduit à une restructuration complète des paysages impliquant la création d'équilibres ou plus exactement d'une succession de déséquilibres nouveaux. La chênaie pure s'est substituée aux forêts mixtes, voire étendue aux dépens de hêtraies ou sapinières à l'équilibre fragile: l'assèchement des versants consécutif à l'intervention humaine est sûrement une des raisons de cette transformation radicale et durable. Tous ces processus passés sont encore discernables de nos jours, révélés par un ensemble contradictoire de phénomènes de déséquilibres et de rééquilibrages.

Cette évolution, discernable dans les Pyrénées française, est différente de la situation des montagnes cantabriques, où la persistance, jusqu'à nos jours, d'une forte tension économique sur la forêt, en dehors de toute réglementation, a amené une surexploitation des chênaies: usage paysan (fabrication d'outils, de charrettes, de tonneaux), usage industriel (combustible pour les locomotives, étayages des mines), etc., les chênaies en bon état y sont devenues très rares (Cervera de Pisuerga).

\section{Une forêt de substitution ?}

Dans les Pyrénées françaises, la situation actuelle de déprise rurale se traduit, pour les chênaies, par des évolutions contradictoires : d'un côté les vieilles chênaies des hauts versants (1 200-1 $700 \mathrm{~m}$ ) tendent à disparaître, alors que de l'autre les peuplements mixtes à chêne s'étendent considérablement dans les zones basses (vers $800-1000 \mathrm{~m}$ ).

\section{La disparition de la chênaie paysanne.}

Incendie et pâturage ont joué un rôle décisif dans la formation et la conservation des chênaies. On pourrait attendre de l'évolution actuelle, marquée par l'abandon des secteurs les moins productifs, comme les soulanes desséchées qui conviennent au chêne, qu'elle se caractérise par la restauration d'équilibres de plus en plus forestiers.

(9) Arch. munic. Sọde, 
En fait, il se produit un curieux phénomène d'hystérésis pastorale, consécutif au déphasage entre pratiques et mode d'exploitation : la baisse de la fréquentation pastorale a entraîné la croissance rapide d'un sous-bois dense, produisant beaucoup de matière sèche (fourrés à Sarothamnus scoparius et Pteridium aquilinum, par ex.), la forme des arbres, qui évolue lentement, restant basse et rabougrie; or, les mises à feu n'ont pas cessé dans ces bois, soit directement, soit en provenance des vacants voisins. Passant dans les sous-bois enfrichés, les incendies prennent une ampleur et une violence beaucoup plus destructrices que par le passé. On est entré aujourd'hui dans un cercle vicieux pouvant conduire à la destruction progressive de la chênaie: plus le sous-bois est dense, plus les feux sont destructeurs pour les arbres, plus le sous-bois est ensoleillé, plus il s'épaissit...

A court ou à moyen terme la forêt disparaît en tant que telle, mais les chênes rejetant sans cesse de souche on aboutit à la création d'une brousse très dense mélangeant cépées de chênes, genêts, fougères, ronces, etc., processus visible en de nombreux secteurs de la soulane de Melles. Les arbres commencent à présenter des blessures de plus en plus profondes puis, après 4 ou 5 incendies successifs, commencent à dépérir. Bien entendu, les hêtres subsistant dans les chênaies sont les premières victimes de cette aggravation de l'incendie; leur disparition à court terme peut êtré considéréc comme significative de l'évolution historique passée des chênaies mixtes, en France comme dans les montagnes cantabriques. Il ne faut cependant pas voir dans cette évolution une destruction brutale comme en forêt méditerranéenne; la résistance des arbrés et la lenteur du phénomène sont attestés par les modifications minimes qu'attestent les comparaisons des photographies aćriennes de Melles entre 1942 et 1982; mais il semble bien que s'accélère aujourd'hui un mouvement, qui ne cessera pas tant que les mises à feu continueront (photos 3 et 4).

Parallèlement à cette dynamique forestière régressive, on assiste aujourd'hui à des évolutions progressives, présageant elles aussi la disparition de la chênaie: la recolonisation par le sapin est très nette partout où il se mélange avec des chênaies (quand celles-ci ne présentent pas des faciès trop secs). C'est le cas de Bareilles, mais aussi de certains bois en vallée d'Oueil : à St-Paul, le bois d'Artigue, sapinière pure, comprend une parcelle de chêne de belle venue; ce petit peuplement, visiblement artificiel, est entièrement recolonisé par une régénération dense de sapin : à moyen terme la chênaie est condamnée.

A Melles les processus sont plus discrets, car entravés par l'incendie. Le sapin a colonisé d'abord les hêtraies qui parsèment la soulane, puis s'étend petit à petit hors de ces refuges humides et imbrûlables; dès qu'il se trouve dans une chênaie, le jeune sapin est à la merci d'un simple feu courant.

Dans la vallée de la Pique, la reconquête du sapin est beaucoup plus spectaculaire. Les hêtraies sont bien sûr les premières touchées par l'envahissement, mais les chênaies (qui montent sur les versants 
est de l'Antenac jusqu'à $1650 \mathrm{~m}$ d'altitude) sont de plus en plus piquetées de sapins voire complètement recolonisćes en sous-bois. La plasticité du sapin est telle qu'il voisine avec les stations de chêne vert de la vallée de la Garonne, au-dessus de Sainte-Marie de Siradan, par exemple.

L'évolution naturelle a été accélérée par l'action des forestiers qui ont contribué à éliminer le chêne au cours du siècle écoulé. En vallée d'Oueil, le bois de Suberdède (Cirès) est décrit en 1880 comme une chênaie mélangée de hêtres (10); aujourd'hui, après un siècle de recépage et de protection, c'est une fûtaie de hêtre en voie d'envahissement par le sapin où l'on n'a plus de chênes que sur les lisières léchées par les écobuages. Les chênaies de Bareilles sont aussi en voie de mutation : coupes à blancs et enrésinements se multiplient, tandis que hêtres et sapins progressent.

\section{La colonisation de l'espace paysan par la chênaie.}

Les vieux peuplements, les seuls existant au XIX* siècle, sont aujourd'hui en plein déséquilibre, sinon en train de disparaître. Pourtant la chênaie est beaucoup plus étendue aujourd'hui qu'il y a un siècle: l'enfrichement spectaculaire, qui est visible dans toutes les zones basses : anciennes prairies ou cultures, pâturages intermédiaires, donne des peuplements mélangés à bouleau, frêne, tilleul, châtaignier, merisier, etc., où le chêne occupe toujours une place importante. Dans les secteurs rocheux, la chênaie peut même être pure. Mais il ne s'agit là que d'un peuplement de transition extrêmement jeunc; la plupart des chênaies qui occupent les bas versants de la vallée de la Pique, par exemple, n'existaient pas voilà un siècle : les archives photographiques en témoignent; on avait à la place un maigre piquetage de chênes buissonnants, rescapés du feu, des chêvres et des coupes (photos 5 et 6).

Ces derniers types d'évolution ne se manifestent pas dans les montagnes cantabriques pour deux raisons principales: d'une part, l'absence de tout résineux (pins ou sapins) fige les dynamiques végétales; d'autre part, et surtout, l'intensité de l'exploitation de la mon. tagne commence à peine à diminuer: le début de la dépopulation ne date que des années 1950, soit un siècle plus tard que dans les Pyrénées françaises. Le paysage est encore sous tension, marquí par une forte occupation humaine actuelle ou peu ancienne, et les évolutions sont trop récentes pour être visibles.

Par contre, les chênaies des Pyrénées françaises apparaissent bien comme un espace en pleine restructuration où les héritages plus ou moins stabilisés voisinent avec des évolutions extrêmement rapides ou des créations récentes. Il semble présomptueux de considérer aujourd'hui ces peuplements comme des stades finaux ou de préjuger. de leur évolution future...

(10) AD 31, 6P38. 
D'un bout à l'autre de la chaîne pyrénéo-cantabrique, la chênaie se présente comme un étonnant témoin des relations entre société montagnarde et végétation forestière : à la fois fossile vivant conservant la marque de pratiques disparues ou d'évolutions très anciennes et avant-garde des mutations en cours. Ni espace défriché par le paysan, ni forêt ordonnée par le forestier, la chênaie paysanne représente un cas rare de sylva organisée comme un saltus pour les besoins des troupeaux tout en étant soumis à des pratiques méticuleuses dignes d'un ager : émondage, soutrage, glanage, etc. Le chêne dans tous ses avatars, seule arbre capable de survivre à un niveau aussi elev'́ de contraintes imposées par l'homme, devient un véritable anthrcpophyte. La chênaiefriche, elle, n'est pas encore forêt, mais n'est déjà plus culture depuis longtemps; les mêmes qualités de résistance, de plasticité, d'adaptivité, qui ont fait du chêne l'arbre le plus humanisé, en font alors le principal indicateur de la déshumanisation de l'espace. 
RÉSUMÉ. - Les chênaies constituent dans les montagnes pyrénéo-cantabriques un révélateur des relations entre forêt et société montagnarde. Elles sont le maillon de l'espace villageois où se sont concentrés les usages les plus variés, tant pastoraux que forestiers. Ces peuplements furent à la fois victimes de l'exploitation traditionnelle (pastoralisme intense, pratique du feu), qui les a profondément remodelés, et soigneusement protégés en tant que réserve de bois indispensable. Aujourd'hui les déséquilibres sont généraux, la chênaie représentant soit un fossile sylvo-pastoral, soit une forme d'enfrichement transitoire.

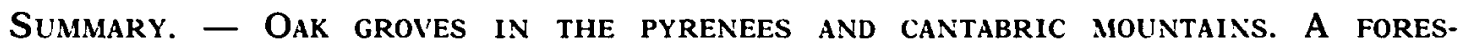
TRY COMPONENT OF THE AGRO-PASTORAL SYSTEM. In the Pyrenees and Cantabric mountains oak woods can be considered as a link between the forest and the mountain society. They are the spot of the village scenery where are seen together diverse uses, as much pastoral as forest, by natives. These plantations are victims of traditional exploitation (intensive pastoralism, firing) which have deeply changed them, whilst being protected as an indispensable wood reserve. Presently disequilibrium is general, the plantations being either a sylvi-pastoral fossil, or a transitory fallow land.

RESUMEN. - LOS ROBLEDALES EN LAS SIERRAS PIRINEO-CANTÁbRICAS : ELEMENTO FORESTAL DEL SISTEMA AGROPASTORIL. En las sierras pirineo-cantábricas, los robledales son un indicador de las relaciones entre el bosque y la sociedad de montaña. Fueron el foco del espacio rural en el que se concentraron los aprovechamientos más diversos, tanto pastoriles como forestales. Víctimas de la explotación tradicional (pastoreo intensivo, quemadas) que los ha alterado profundamente, a la vez fueron protegidos cuidadosamente como inidspensable reserva de madera. Hoy los desequilibrios dominan: el robledal aparece sea como un fosil silvopastoril, sea como una forma transitoria hacia el yermo.

Mots-Clés. - Pyrénées, Montagnes cantabriques, Foré, histoire des forêts, agro-sylvo-pastoral, feu, pastoral, écobuage, incendie de forêts, enfrichement, $X I X^{*}$ et $X X^{*}$ s.. 